\title{
Clinical Neurophysiological and Automated EEG-Based Diagnosis of the Alzheimer's Disease
}

\author{
Shreya Bhat ${ }^{a}$ U. Rajendra Acharyaa ${ }^{b, c}$ Nahid Dadmehr ${ }^{d}$ Hojjat Adeli ${ }^{\mathrm{e}-\mathrm{j}}$ \\ a Department of Psychiatry, St. John's Research Institute, Bangalore, India; ${ }^{b}$ Department of Electronics and \\ Computer Engineering, Ngee Ann Polytechnic, Singapore, Singapore; ' Department of Biomedical Engineering, \\ Faculty of Engineering, University of Malaya, Kuala Lumpur, Malaysia; ${ }^{\mathrm{d} D i v i s i o n}$ of Neurology, Mt. Carmel Hospitals, \\ and Departments of ${ }^{\mathrm{e}}$ Neuroscience, ${ }^{\mathrm{f} B i o m e d i c a l ~ E n g i n e e r i n g, ~}{ }^{\mathrm{g} B i o m e d i c a l ~ I n f o r m a t i c s,}{ }^{\text {h}}$ Electrical and Computer \\ Engineering, 'Civil, Environmental, and Geodetic Engineering and ${ }^{j}$ Neurology, The Ohio State University Columbus, \\ Columbus, Ohio, USA
}

\section{Key Words}

Alzheimer's · Plaques · Tangles · Donepezil · Apraxia ·

Neurodegeneration · Aphasia

\begin{abstract}
Alzheimer's disease (AD) is a progressive disorder affecting intellectual, behavioral and functional abilities. It is associated with age and pathological alterations leading to the formation of amyloid plaques and tangles. It is the most common source of dementia in the older population, which varies in its degrees of severity. We are yet to find efficient methods of diagnosis of $A D$, as its symptoms vary among individuals. This paper presents a review of recent research on the clinical neurophysiological and automated electroencephalography-based diagnosis of the AD. Various therapeutic measures are also discussed briefly.
\end{abstract}

C 2015 S. Karger AG, Basel

\section{Introduction}

Senescence is defined as the normal aging process where the human body undergoes several physiological changes. It affects cognition, thought process, learning ability, and emotions and reduces the tempo of one's daily activities. Aging is not pathology but a normal physiological change leading to memory disorders. Dementia is a neurological disorder characterized by memory, language and cognitive impairment and loss in behavioral abilities. It is caused by brain cell death and worsens with age but is not linked to the typical aging process $[1,2]$. Other potential causes of dementia are head injury, brain tumor, stroke, cerebral nervous system infection, vitamin deficiency, alcohol, exposure to heavy metals, and so on. Alzheimer's disease (AD) is the common basis of dementia in the elderly population [3].

$\mathrm{AD}$ is a progressive brain cell death and an irreversible process that happens over a course of time resulting in memory loss, inability to learn new things and perform calculations, unbalanced perception of space, depression, delusions and cognitive decline. These impairments hamper patients' social functioning and behavioral abilities $[4,5]$. Cera et al. [6] studied the effect of AD on different cognitive functions such as orientation, language, attention, praxis, visual perception and executive function. They report that severity of language impairment and orofacial apraxias in the individuals with $\mathrm{AD}$ is directly proportional to the gradual progression of $\mathrm{AD}$. The clinical features of AD are summarized in table 1.

\section{KARGER 125}

(C) 2015 S. Karger AG, Base

0014-3022/15/0744-0202\$39.50/0 
Table 1. Main features of AD [98-100]

Clinical features of $\mathrm{AD}$

\begin{tabular}{ll}
\hline Memory deficit & $\begin{array}{l}\text { Learning disability } \\
\text { Aphasia }\end{array}$ \\
Language impairment affecting speech and \\
information processing \\
Agraxia & $\begin{array}{l}\text { Motor disorder } \\
\text { Inability to recognize and identify people or } \\
\text { objects }\end{array}$ \\
\hline
\end{tabular}

Table 2. Different stages of AD [18]

\begin{tabular}{ll}
\hline Stages of AD & Symptoms of AD \\
\hline Mild AD & $\begin{array}{l}\text { Memory loss, poor judgment } \\
\text { Mood swings, repetitive questions } \\
\text { Difficulty in doing mathematical calculations }\end{array}$ \\
\hline Moderate AD & $\begin{array}{l}\text { Unable to learn new things } \\
\text { Difficulty in recognizing family members and } \\
\text { friends } \\
\text { Possesses hallucinations, delusions, paranoia } \\
\text { and impulsive behavior }\end{array}$ \\
\hline Severe AD & Dependent and bedridden \\
\hline
\end{tabular}

$\mathrm{AD}$ is the sixth leading cause of death in the United States [7]. Patients die due to fall, malnutrition, dehydration, traumatic brain injury, and complications such as ab-ingestis pneumonia, pneumonia, bladder infection, and diabetes. $\mathrm{AD}$ often becomes fatal within 10 years of its commencement. According to the Alzheimer's Association, $13 \%$ of people over 65 years and $45 \%$ of people over 85 years have $\mathrm{AD}$ [8]. Some of the potential risk factors associated with $\mathrm{AD}$ are age, inheriting apolipoprotein E (APOE) gene [9] and sleep apnea [10-12]. Females are more prone to $\mathrm{AD}$ than males. $\mathrm{AD}$ progresses in prevalence after the age of 65 . Prevalence rate increases by 2 -fold every 5 years between the ages of 65 and 95 . It rises from $\sim 2 \%$ in those aged $65-69,4 \%$ in $70-74,8 \%$ in $75-79,16 \%$ in $80-85$ and $\sim 35-40 \%$ over the age of 85 [13]. Neuro-researchers are trying to analyze possible age-related changes in the $\mathrm{AD}$ brain. Some of these changes include inflammation of the brain, atrophy, mitochondrial dysfunction and production of unstable molecules in the brain [13]. AD can also be inherited. Individuals with primary relative having AD show a 3-4fold increase in AD risk and it increases to a fold of 7.5 in individuals who have two or more primary relatives with $\mathrm{AD}[14]$.

EEG-Based Diagnosis of the AD
Early onset of $\mathrm{AD}$ is seen in people between 30 and 60 years of age due to inherited genes in an autosomal-dominant manner $[15,16]$. The younger population with early-onset $\mathrm{AD}$ possesses a distinct cognitive profile reflecting different distribution of underlying neuropathology [17]. Ossenkoppele et al. [17] observed that increased amyloid-beta $(\mathrm{A} \beta)$ deposition in the parietal lobe and metabolic dysfunction contribute to the distinct cognitive profile of patient's with AD.

AD is broadly classified into 3 stages [18] listed in table 2. AD commences with signs of mild cognitive impairment (MCI) [19]. Individuals with MCI have significantly high memory loss compared to individuals with normal aging but possess normal behavioral abilities. As AD progresses, memory loss worsens and leads to mild $\mathrm{AD}$ characterized by poor judgmental ability, emotionally disturbed mind frame and inability to perform simple calculations [18].

Moderate $\mathrm{AD}$ is the second stage of $\mathrm{AD}$ where sensory processing and conscious thought process are hampered. Individuals with moderate $\mathrm{AD}$ experience hallucinations, delusions, paranoia and impulsive behavior are unable to recognize their kith and kin. The third stage of AD leads to severe symptoms resulting in cognition impairment, behavioral disabilities and atrophy when the patient becomes completely dependent on others [18].

After the detection of early symptoms of AD (mild $\mathrm{AD}$ ) as indicated in table 2, a neurologist conducts a physical examination and differential diagnosis in order to dismiss other potential diseases or neurological disorders [7, 13]. These examinations include thyroid and vitamin B12 deficiency tests. After ascertaining that the memory loss is not due to hormonal deficiencies, clinicians recommend the brain-imaging technique. Generally, CT and MRI are used to identify the structural changes in the brain [7, 13]. Single photon emission computerized tomography and photon emission tomography (PET) can detect activities like blood flow or metabolism in the brain. In recent years, researchers have attempted to develop computer algorithms for automated electroencephalography (EEG)-based diagnosis of the $\mathrm{AD}$ and their precision for detection of $\mathrm{AD}$ is being evaluated $[4,10,38$, $75,76]$.

Section 2 provides a brief overview of the pathology of AD. Section 3 describes the layout of this review, which is the latest research on the early diagnosis of AD including the application of the EEG signal analysis. Various therapeutic measures are discussed in Section 4 . Section 5 concludes the paper. 


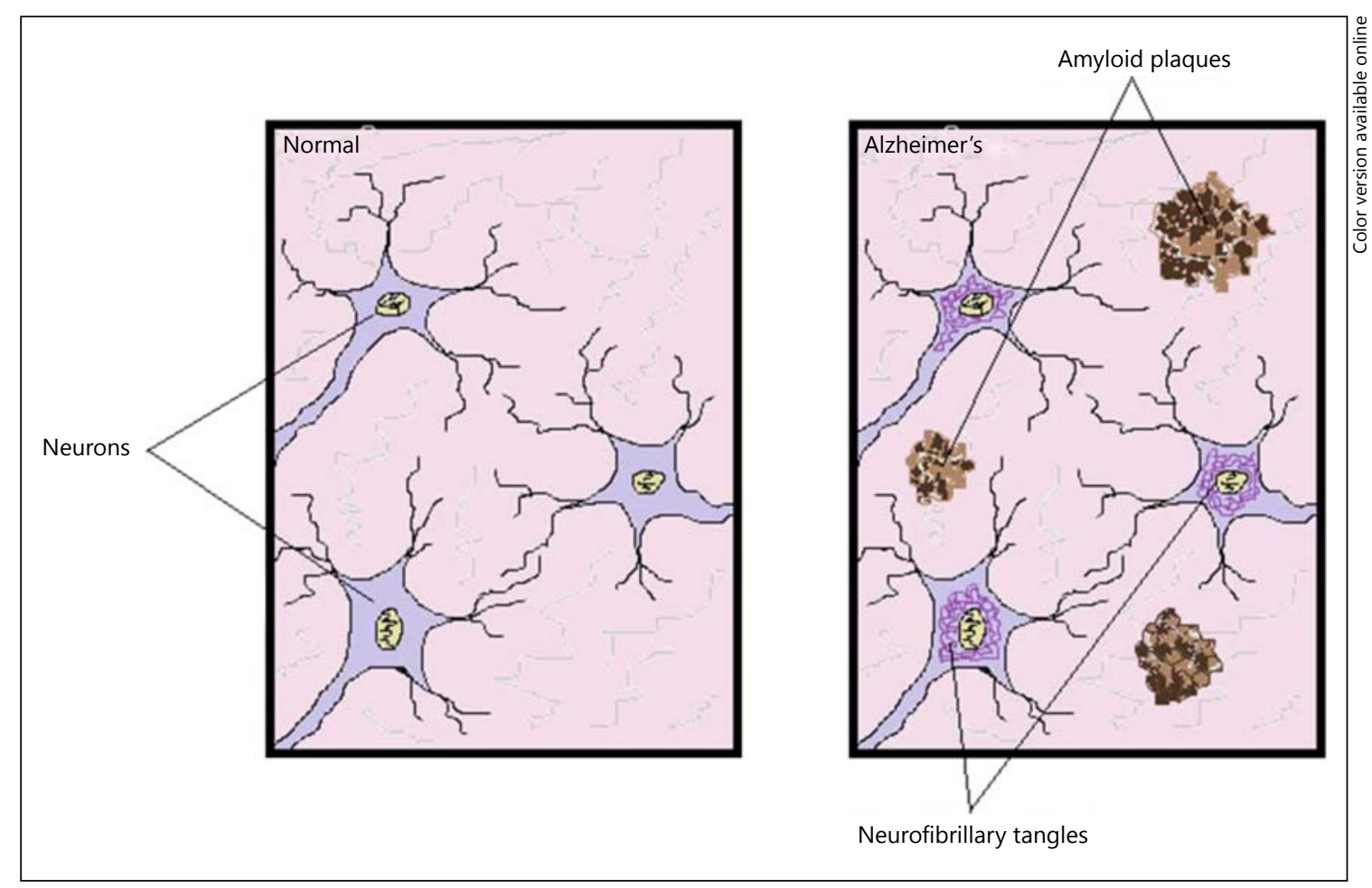

Fig. 1. Pathological alterations resulting in the formation of plaques and tangles.

\section{Pathology and Pathogenesis of AD}

The main pathological alterations observed in the $\mathrm{AD}$ brain are neuritic plaques and neurofibrillary tangles (fig. 1). They are tiny inclusions, abnormal protein clumps found in the $\mathrm{AD}$ brain tissue during autopsy. Plaques are formed by the accumulation of a protein called beta-amyloid between the dying brain cells. These plaques are globular microscopic lesions composed of extracellular $\mathrm{A} \beta$ in the core surrounded by degenerating axonal endings. The degeneration of a protein called tau gives rise to tangles that are fibrillary, cytoplasmatic structures within the neurons [20-23]. Neuropil threads, amyloid angiopathy and granulovacuolar degeneration are some of the pathological features observed in the $\mathrm{AD}$ brain [5].

Bie et al. [24] found that amyloid-induced neuroinflammation leads to epigenetic suppression [25] of NLGN1 (neuroligin1) expression and microglia-mediated inflammation may advance the activity of transcriptional receptors damaging central glutamatergic synapses and memory in the brain. Lunnon et al. [26] analyzed methylomic variation in $\mathrm{AD}$ using samples from four human postmortem brain cohorts. They observed differentially methylated region in the ANK1 (ankyrin1) gene
[27] associated with the superior temporal gyrus and prefrontal cortex (entorhinal cortex), a primary symptom of AD.

Abnormal protein deposition spreads to hippocampus leading to memory loss. As more neurons die, the brain regions shrink, resulting in atrophy. The progression of neuronal vulnerability is related to the morphological and biochemical characteristics of AD. The number of nerve cells in the brain tissue progressively decreases and the neuronal connectivity weakens [28]. The total brain size shrinks due to the degeneration of nerve cells [29].

Neurodegeneration of hippocampus and the variation of resting eyes-closed EEG signals are major features observed in MCI and AD [30]. The progressive atrophy of hippocampus is also correlated with the reduction of cortical alpha power [30]. Migliaccio et al. [31] mapped the succession of gray matter atrophy in early-onset $\mathrm{AD}$ and late-onset $\mathrm{AD}$. MRI scans of patients who met $\mathrm{AD}$ criterion and who showed $\mathrm{AD}$ symptoms before the age of 65 were obtained. Voxel and tensor-based morphometry [32] is used for diagnosis. They reported that medial temporal or lateral neocortical regions underwent atrophy in the beginning and $\mathrm{AD}$ progressed to medial parietal cortices and medial precuneus regions in the later stage. 
Madeira et al. [33] measured D-serine levels in the postmortem hippocampal, cortical samples and cerebrospinal fluid (CSF) from the control group and subjects with AD. They observed difference in the brain and CSF $\mathrm{D}$-serine levels of $\mathrm{AD}$ and control group. D-Serine levels in parietal cortex, hippocampus and CSF were higher in individuals with $\mathrm{AD}$ than the control group. Some of the neurochemical changes associated with $\mathrm{AD}$ are reduction in cholinergic activity, insufficiency in glutamate, serotonin, somatostatin, norepinephrine, and corticotrophin-releasing factors [34].

Neuroglial cells are a group of heterogeneous cells responsible for neuroprotection and defense against exogenous and endogenous entities. At the onset of neurodegenerative disorders, neuroglial cells become asthenic and lose their neuroprotective, defensive and homeostatic capabilities. It is found that paralytic neuroglial cells are responsible for the progression of $\mathrm{AD}$ [35].

Goll et al. [36] demonstrated that auditory scene analysis is susceptible to the neurodegenerative process in AD. Auditory scene analysis describes the processing of vocal and non-vocal sounds. Neuroanatomical changes in auditory scene analysis are identified in posterior corti$\mathrm{cal}$ areas. They found that $\mathrm{AD}$ is linked to the primary and generic disruption of the auditory scene analysis.

\section{Clinical Neurophysiological Diagnosis of AD}

In preclinical $\mathrm{AD}$, the pathology of $\mathrm{AD}$ represents an insidious process that starts years before the occurrence of clinical manifestations. Early diagnosis of $\mathrm{AD}$ and need for its biomarkers becomes challenging because of the delay between the pathological and behavioral symptoms $[21,37]$. Neurologists, neuro-scientists and neuro-researchers are all involved in the Alzheimer's research [38, 39] for its early intervention, to predict the rate of cognitive decline and to measure the impact of drug treatments.

Mattsson et al. [40] studied the correlation between $\mathrm{CSF}$ and PET A $\beta$ in AD. They observe that reduction in CSF and increase in PET A $\beta$ is strongly related to earlystage $\mathrm{AD}$ and the disease progression respectively. Hence, they concluded that these parameters can be considered the biomarkers of $\mathrm{AD}$.

Using PET for diagnosis of AD, Viola et al. [41] found that $A \beta$ oligomers are responsible for memory loss in patients with $\mathrm{AD}$. The oligomer-specific antibodies, binding to $A \beta$ oligomers, are induced into brain tissues and cells. They can generate MRI signals that help in identify- ing the early-stage $\mathrm{AD}$. The magnetic nanostructures targeting neurotoxic $A \beta$ oligomers can also provide feedback on the effects of antioxidants.

Moyse et al. [42] study the concept of perception processing in social cognition among AD patients. They evaluated the impairment of facial-age estimation in patients with mild and moderate AD. They observed individuals with mild $\mathrm{AD}$-faced difficulties in estimating the age of middle-aged adults, whereas individuals with moderate $\mathrm{AD}$ had difficulties in calculating the age of young adults. However, it is surprising to find individuals with $\mathrm{AD}$ accurately assessing the age of older adult faces compared to the control group.

Oleson and Murphy [43] reported ApoE $\varepsilon 4$ gene as the major risk factor of $\mathrm{AD}$ associated with olfactory and cognitive deficits. Individuals carrying more than one allele of ApoE gene $(\varepsilon 4 / 4)$ are at greater risk of developing $\mathrm{AD}$. Olfactory functioning was examined in homozygous $\varepsilon 4 / 4$ adults with probable AD. These individuals showed deficits in odor memory and identification compared to individuals with heterogeneous $\varepsilon 3 / 4$ ApoE carriers. Thus, the odor tasks may prove to be helpful in the early diagnosis of AD.

Lopez-de-Ipina et al. [44] discuss a noninvasive methodology for early diagnosis of AD based on speech analysis and emotional temperature. Speech features such as spontaneous speech and emotional response are extracted from the suspected AD patients. Machine learning algorithms [45-47] are applied to the significant features, and the severity of $\mathrm{AD}$ is evaluated.

\section{EEG-Based Diagnosis of AD}

The advent of neuroimaging techniques such as EEG $[48,49]$, magnetoencephalography $[19,50]$, MRI [51, 52], functional MRI (fMRI) [53, 54], structural MRI (sMRI) have increased the study of cognitive neuroscience. They facilitate the noninvasive research of human brain mechanism and study of its complex connectivity $[55,56]$. MRI and fMRI [57], however, are costly and not clinically valuable at the onset of $\mathrm{AD}$. On the other hand, EEG is a noninvasive and cost-effective technique with a potential to detect $\mathrm{AD}$ in its early stages.

The EEG signals are highly nonlinear in nature. Variation in the EEG signals cannot be deciphered by mere ocular assessment. Earlier, Smith [58] reported that EEG signals are normal during the early stage of $\mathrm{AD}$, but as the disease progresses, alpha waves disappear and slow waves become more apparent. In some cases, periodic sharp 
waves are also visible but minute variations occurring in the EEG signals during the early onset of AD remains undetermined. Computer-aided diagnostic tools can be used to identify the subtle variations in the EEG signals [59-61]. Time-domain, frequency domain, time-frequency analysis $[62,63]$ and nonlinear methods can be used to analyze the EEG signals. In recent years, Adeli and associates $[48,64,65]$ have advanced the idea that adroit integration of three computing paradigms, time-frequency signal processing [66-68], chaos theory and nonlinear methods $[69,70]$, and pattern recognition techniques such as artificial neural networks [71-73] is the best approach to analyze nonstationary and highly chaotic signals. Significant features can be extracted by nonlinear dynamics and classified using different data-mining techniques and neural networks [74].

Adeli et al. [75] presented a review of research on computational modeling of $\mathrm{AD}$ employing computer imaging, classification models, connectionist neural models, and biophysical neural models up to 2005. Adeli et al. [76] summarized the early efforts on the use of EEG for diagnosis of AD. Adeli et al. [62] present a spatiotemporal wavelet-chaos methodology for analysis of EEGs and their delta, theta, alpha, and beta sub-bands for the purpose of discovering potential markers of AD. The nonlinear dynamics of the EEG and EEG sub-bands are quantified employing the correlation dimension representing system complexity, and the largest Lyapunov exponent representing system chaoticity. They report potential markers for the diagnosis of AD using a sample of 20 subjects diagnosed with probable $\mathrm{AD}$ and 7 controls.

Dauwels et al. [77] report that slowing of the EEG in patients with $\mathrm{AD}$, reduced complexity [78], and perturbations in EEG synchrony [79] are the major effects in Alzheimer's EEG signals. Trambaiolli et al. [80] apply support vector machine (SVM) [81] to identify significant patterns in Alzheimer's and normal EEG epochs. A quantitative EEG-processing method is used to differentiate between $\mathrm{AD}$ and normal patients. A classification accuracy of $87 \%$ and sensitivity of $91.7 \%$ is obtained using SVM.

Sankari et al. [82] investigate interhemispheric, intrahemispheric and distal coherence in individuals with AD. The EEG electrode coherence is computed over all the frequency bands. An increase in the characteristic pattern of the left intrahemispheric frontal coherence in the alpha, theta, delta frequency bands, left intrahemispheric temporo-parietal coherence in all bands and a decrease in the right temporo-parieto-central coherence in all bands is observed in patients with $\mathrm{AD}$, whereas an increase in left temporo-parietal, temporo-central and frontal coherence in specific bands implied progression of $\mathrm{AD}$ leading to loss of memory and cognitive decline.

Using the concept of fractality dimension (FD) Ahmadlou et al. [63] present a wavelet-chaos methodology for EEG-based diagnosis of AD. They determine the most discriminative FD and the corresponding loci and EEG sub-bands for discriminating between $\mathrm{AD}$ and healthy subjects. They report an accuracy of $99.3 \%$ for diagnosis of the AD based on Katz FD using a sample of 20 subjects diagnosed with probable $\mathrm{AD}$ and 7 controls.

Yang et al. [83] applied multiscale entropy (MSE) [84] to identify the variations in the AD EEG signals. Consistency of MSE measures in different EEG epochs and their correlation to cognitive and neuropsychiatric symptoms is assessed. They report that decreased MSE complexity measured by short-time scales and increased MSE complexity measured by long-time scales are associated with increased severity of $\mathrm{AD}$, and the MSE complexity of temporal and occipitoparietal regions is related to cognitive and neuropsychiatric manifestations. Al-Jumeily et al. [85] compare EEG signals of left and right temporal regions of brain with the rest of the brain regions and report reduced synchronization in Alzheimer's EEG signals compared to normal EEG signals.

Labate et al. [86] measure the EEG signal complexity to predict the disease progression and also to differentiate brain states that are proportional to the varying stages of AD. They applied three entropic complexity measures (permutation entropy, Lempel-Ziv complexity, sample entropy) to the EEG signals and performed a multi-scale multivariate analysis. They observe significant interchannel correlation among the EEG channels and conclude that the severity of the disease is prominent in the EEG dynamic complexity and that EEG is a potentially useful tool for early diagnosis of AD.

Waser et al. [87] study univariate and multivariate spectral densities to measure slowing and decreased synchrony in the EEG signals. Coherences, partial coherences, bivariate Granger causality and conditional Granger causality are analyzed to measure EEG synchrony and spectral power in the predefined frequency bands is calculated to measure signal slowing. These measures are computed for resting-state EEG signals for subjects with AD. They conclude that automated analysis of variations in the EEG signals of individuals with AD may contribute to the medical diagnosis of the disease.

The amplitude of alpha rhythms is related to AD neurodegeneration along with pathologic aging. Babiloni et al. [88] observed abnormality in alpha rhythms obtained 
by occipital sources of resting-state EEG in patients with amnesic MCI and AD. Gallego-Jutgla et al. [89] analyze changes in EEG synchrony in the theta band and observe an increase in the EEG synchrony in its narrow frequency ranges. They also propose a synchrony ratio to enhance the difference between subjects with $\mathrm{AD}$ and control group.

McBride et al. [90] use scalp EEG-based causality measurements and perform Sugihara causality analysis [91] to differentiate among normal aging, $\mathrm{MCI}$ and $\mathrm{AD}$ patients as these measurements have different distribution for different cognitive groups. A reconstruction model for each EEG channel is developed to predict the signal in the current channel using data of the remaining channels. The leave-one-out principle is used for training and Sugihara causality between different channels is described by a quality score obtained by comparing the reconstructed and original signals. They report an average classification accuracy of $96.5 \%$ after a 3-way classification.

\section{Therapeutic Interventions}

AD cannot be cured, but measures can be developed to delay its symptoms and help the $\mathrm{AD}$ population live with ease. Enhanced and efficient care quality and public awareness on $\mathrm{AD}$ can support people with $\mathrm{AD}$ and their families. Current AD treatments are classified as neuroprotective approach using antioxidants [92] and symptomatic approach based on the enhancement of neurotransmitter system.

Drugs such as donepezil, alantamine, rivastigmine, and tacrinecan are used as acetylcholinesterase inhibitor [93] that enhances cholinergic system of the brain. Acetylcholine plays a major role in the manifestation of cognitive and behavioral symptoms in $\mathrm{AD}$ [94]. The latest addition for treatment of $\mathrm{AD}$ is memantine that blocks $\mathrm{N}$-methyl-D-aspartate receptor receptors and acts on the glutamatergic system. Ahnaou et al. [95] studied the effects of cognition enhancers such as donepezil, galantamine, tacrine on the EEG oscillations for cortical and subcortical networks. These enhancers help in the systematic development of cortical slow theta and gamma oscillations. Improved neuronal connectivity revealed elevated coherent slow theta activity in parieto-occipital and in between interhemispheric cortical areas. The study concluded that therapeutic drugs such as donepezil, galantamine, and rivastigmine have cognition-enhancing potential that can delay the commencement of $\mathrm{AD}$.

EEG-Based Diagnosis of the AD
Blurton-Jones et al. [96] found that neural stem cell transplantation enhanced synaptic connectivity and improved cognition in AD patients. Stem cells of AD patients are genetically modified and implanted in their brain hallmarks. They observed that neural connectivity between the brain cells increased, whereas accumulated A $\beta$ protein degenerated. Modified stem cells increased the level of neprilysin, an enzyme that breaks down $A \beta$ and lowers its activity in the AD brain. Chancellor et al. [97] explored the art therapy on AD patients and observed that it improved social behavior, self-respect and neuropsychiatric symptoms. Immunization therapy, cognitive training and physical activities are some of the therapeutic measures applied on subjects with AD. Effects of cardiovascular and diabetes treatments on $\mathrm{AD}$ patients are also being explored.

\section{Conclusion}

$\mathrm{AD}$ is a neurocognitive disorder that progresses after its commencement in the older population. It is characterized by behavioral variability, cognitive decline, depression, delusions and memory loss. Studies reveal that formation of neuritic plaques, tangles, neuropil threads, granulovacuolar degeneration and amyloid angiopathy are some of the pathological variations causing AD. Neuroprotective and symptomatic approaches such as antioxidants and neurotransmitters have proved to be effective in the treatment of $\mathrm{AD}$ symptoms and found to delay their development. The paragraph has been simplified as follows: there are no treatments that can cure $\mathrm{AD}$, but drugs have been developed and continue to be developed that can treat symptoms of the disease and delay its progression. As such, early diagnosis is the key in the treatment of the disease. Advances in neuroimaging technology, cognitive neuroscience, psychopathology, neuropathology and neurobiology are leading to the discovery of $\mathrm{AD}$ biomarkers for its early diagnosis. Researchers are also working on refining and improving the accuracy of the EEG-based diagnosis of $\mathrm{AD}$ [101]. The dream research and discovery is to develop an algorithm for early onset diagnosis of the $\mathrm{AD}$ but that will be sometime in the future.

\section{Disclosure Statement}

None of the authors have any personal or financial conflict of interest that could unsuitably influence the writing or publication of this manuscript. 


\section{References}

1 Grady C: The cognitive neuroscience of ageing. Nat Rev Neurosci 2012;13:491-505.

2 MNT-Medical News Today: What is Dementia? The Signs, Symptoms and Causes of Dementia. http://www.medicalnewstoday.com/ articles/142214.php (accessed January 16, 2015).

3 McKhann GM, Knopman DS, Chertkow H, Hyman BT, Jack CR Jr, et al: The diagnosis of dementia due to Alzheimer's disease: recommendations from the national institute on aging-Alzheimer's association workgroups on diagnostic guidelines for Alzheimer's disease. Alzheimers Dement 2011;7:263-269.

4 Pressman P, Rabinovici GD: Alzheimer's Disease; in R.B. Baroff, M.J. Aminoff (eds.): Encyclopaedia of the Neurological Sciences, New York, Elsevier, 2014, Vol 1, pp 122-127.

5 MacGill M: What is Alzheimer's Disease? Cause, Symptoms and Treatment. 2014. http:// www.medicalnewstoday.com/articles/159442. php (accessed January 16, 2015).

6 Cera ML, Ortiz KZ, Bertolucci PH, Minett TS: Speech and orofacial apraxias in Alzheimer's disease. Int Psychogeriatr 2013;25:1679-1685.

7 Alzheimers.net: Alzheimer's Statistics. 2015. http://www.alzheimers.net/resources/ alzheimers-statistics/ (accessed May 24, 2015).

8 Agamanolis DP: Degenerative Diseases. Neuropathology. http://neuropathology-web. org/chapter9/chapter9bAD.html (accessed January 20, 2015).

9 Chouraki V, Seshadri S: Genetics of Alzheimer's disease. Adv Genet 2014;87:245-294.

10 Hot P, Rauchs G, Bertran F, Denise P, Desgranges $\mathrm{B}$, et al: Changes in sleep theta rhythm are related to episodic memory impairment in early Alzheimer's disease. Biol Psychol 2011; 87:334-339.

11 Pan W, Kastin AJ: Can sleep apnea cause Alzheimer's disease? Neurosci Biobehav Rev 2014;47:656-669.

12 Peter-Derex L, Yammine P, Bastuji H, Croisile B: Sleep and Alzheimer's disease. Sleep Med Rev 2015;19:29-38.

13 NIH-Alzheimer's Disease Fact Sheet, National Institute of Aging. 2015. http://www.nia. nih.gov/alzheimers/publication/alzheimersdisease-fact-sheet (accessed January 29, 2015).

14 Gao S, Hendrie HC, Hall KS, Hui S: The relationships between age, sex, and the incidence of dementia and Alzheimer disease: a metaanalysis. Arch Gen Psychiatry 1998;55:809815.

$15 \mathrm{Wu}$ L, Rosa-Neto P, Hsiung GY, Sadovnick $\mathrm{AD}$, Masellis M, et al: Early-onset familial Alzheimer's disease (EOFAD). J Neurol Sci 2012;39:436-445.

16 DeFina PA, Moser RS, Glenn M, Lichtenstein JD, Fellus J: Alzheimer's disease clinical and research update for health care practitioners. J Aging Res 2013;2013:207178.

17 Ossenkoppele R, Zwan MD, Tolboom N, van Assema DM, Adriaanse SF, et al: Amyloid burden and metabolic function in early-onset Alzheimer's disease: parietal lobe involvement. Brain 2012;135(pt 7):2115-2125.

18 Hyman BT, Phelps CH, Beach TG, Bigio EH, Cairns NJ, et al: National institute on aging Alzheimer's association guidelines for the neuropathologic assessment of Alzheimer's disease. Alzheimers Dement 2012;8:1-13.

19 Ahmadlou M, Adeli A, Bajo R, Adeli H: Complexity of functional connectivity networks in mild cognitive impairment subjects during a working memory task. Clin Neurophysiol 2014;125:694-702.

20 Thathiah A, De Strooper B: The role of G protein-coupled receptors in the pathology of Alzheimer's disease. Nat Rev Neurosci 2011;12: 73-87.

21 Gregoria PG, Pancorbo RY: Preclinical Alzheimer's disease. Rev Clin Gerontol 2014;24: 117-121.

22 Reitz C, Mayeux R: Alzheimer disease: epidemiology, diagnostic criteria, risk factors and biomarkers. Biochem Pharmacol 2014;88: 640-651.

23 Herukka SK, Rummukainen J, Ihalainen J, von Und Zu Fraunberg M, Koivisto AM, et al: Amyloid- $\beta$ and tau dynamics in human brain interstitial fluid in patients with suspected normal pressure hydrocephalus. J Alzheimers Dis 2015;46:261-269.

24 Bie B, Wu J, Yang H, Xu JJ, Brown DL, Naguib $\mathrm{M}$ : Epigenetic suppression of neuroligin 1 underlies amyloid-induced memory deficiency. Nat Neurosci 2014;17:223-231.

25 Lord J, Cruchaga C: The epigenetic landscape of Alzheimer's disease. Nat Neurosci 2014;17: 1138-1140.

26 Lunnon K, Smith R, Hannon E, Jager PL, Srivastava $\mathrm{G}$, et al: Methylomic profiling implicates cortical deregulation of ANK1 in Alzheimer's disease. Nat Neurosci 2014;17: 1164-1170.

27 Jager PL, Srivastava G, Lunnon K, Burgess J, Schalkwyk LC, et al: Alzheimer's disease: early alterations in brain DNA methylation at ANK1, BIN1, RHBDF2 and other loci. Nat Neurosci 2014;17:1156-1163.

28 D'Amelio M, Rossini PM: Brain excitability and connectivity of neuronal assemblies in Alzheimer's disease: from animal models to human findings. Prog Neurobiol 2012;99:42-60.

29 He Y, Chen Z, Gong G, Evans A: Neuronal networks in Alzheimer's disease. Neuroscientist 2009; 15:333-350.

30 Babiloni C, Frisoni GB, Pievani M, Vecchio F, Lizio R, et al: Hippocampal volume and cortical sources of EEG alpha rhythms in mild cognitive impairment and Alzheimer disease. NeuroImage 2009;44:123-135.

31 Migliaccio R, Agosta F, Possin KL, Canu E, Filippi M, et al: Mapping the progression of atrophy in early- and late-onset Alzheimer's disease. J Alzheimers Dis 2015;46:351-364.

32 Ghosh-Dastidar S, Adeli H, Dadmehr $\mathrm{N}$ : Voxel-based morphometry in Alzheim- er's patients. J Alzheimers Dis 2006;10:445447.

33 Madeira C, Lourenco MV, Vargas-Lopes C, Suemoto CK, Brandão CO, et al: D-serine levels in Alzheimer's disease: implications for novel biomarker development. Transl Psychiatry 2015;5:e561.

34 Yaari R, Corey-Bloom J: Alzheimer's disease. Semin Neurol 2007;27:32-41.

35 Verkhratsky A, Marutle A, Rodríguez-Arellano JJ, Nordberg A: Glial asthenia and functional paralysis: a new perspective on neurodegeneration and Alzheimer's disease. Neuroscientist 2014;pii:1073858414547132.

36 Goll JC, Kim LG, Ridgway GR, Hailstone JC, Lehmann $\mathrm{M}$, et al: Impairments of auditory scene analysis in Alzheimer's disease. Brain 2012;135(pt 1):190-200.

37 Wurtman R: Biomarkers in the diagnosis and management of Alzheimer's disease. Metabolism 2015;64(3 suppl 1):S47-S50.

38 Ahmadlou M, Adeli H, Adeli A: New diagnostic EEG markers of the Alzheimer's disease using visibility graph. J Neural Transm (Vienna) 2010;117:1099-1109.

39 Moretti DV: Scalp EEG markers in subjects with cognitive impairment and Alzheimer's disease. Int J Psycho 2012;85:350-351.

40 Mattsson N, Insel PS, Donohue M, Landau S, Jagust WJ, et al: Independent information from cerebrospinal fluid amyloid- $\beta$ and florbetapir imaging in Alzheimer's disease. Brain 2015;138(pt 3):772-783.

41 Viola KL, Sbarboro J, Sureka R, De M, Bicca $\mathrm{MA}$, et al: Towards non-invasive diagnostic imaging of early-stage Alzheimer's disease. Nat Nanotechnol 2015;10:91-98.

42 Moyse E, Bastin C, Salmon E, Brédart S: Impairment of age estimation from faces in $\mathrm{Al}$ zheimer's disease. J Alzheimers Dis 2015;45: 631-638.

43 Oleson S, Murphy C: Olfactory dysfunction in ApoE \&4/4 homozygotes with Alzheimer's disease. J Alzheimers Dis 2015;46:791-803.

44 López-de-Ipiña K, Alonso JB, Solé-Casals J, Barroso N, Henriquez $\mathrm{P}$, et al: On automatic diagnosis of Alzheimer's disease based on spontaneous speech analysis and emotional temperature. Cogn Comput 2015;7:44-55.

45 Gurubel KJ, Alanis AY, Sanchez EN, CarlosHernandez S: A neural observer with timevarying learning rate: analysis and applications. Int J Neural Syst 2014;24:1450011.

46 Huo J, Gao Y, Yang W, Yin H: Multi-instance dictionary learning for detecting abnormal events in surveillance videos. Int J Neural Syst 2014;24:1430010.

47 Friedrich J, Urbanczik R, Senn W: Code-specific learning rules improve action selection by populations of spiking neurons. Int J Neural Syst 2014;24:1450002.

48 Adeli H, Ghosh-Dastidar S: Automated EEGBased Diagnosis of Neurological Disorders Inventing the Future of Neurology. Boca Raton, CRC Press, Taylor \& Francis, 2010. 
49 Kwon M, Kavuri S, Lee M: Action-perception cycle learning for incremental emotion recognition in a movie clip using 3D fuzzy GIST based on visual and EEG signals. Integr Comput Aided Eng 2014;21:295-310.

50 Hulbert S, Adeli H: EEG/MEG- and imagingbased diagnosis of Alzheimer's disease. Rev Neurosci 2013;24:563-576.

51 Perez G, Conci A, Moreno AB, HernandezTamames JA: Rician noise attenuation in the wavelet packet transformed domain for brain MRI. Integr Comput Aided Eng 2014;21:163175.

52 Gonçalves N, Nikkilä J, Vigário R: Self-supervised MRI tissue segmentation by discriminative clustering. Int J Neural Sys 2014;24: 1450004.

53 Hampel H, Prvulovic D, Teipel SJ, Bokde AL: Recent developments of functional magnetic resonance imaging research for drug development in Alzheimer's disease. Prog Neurobio 2011;95:570-578.

54 Prvulovic D, Bokde AL, Faltraco F, Hampel $\mathrm{H}$ : Functional magnetic resonance imaging as a dynamic candidate biomarker for Alzheimer's disease. Prog Neurobiol 2011;95:557569.

55 Laske C, Sohrabi HR, Frost SM, López-de-Ipiña $\mathrm{K}$, Garrard P, et al: Innovative diagnostic tools for early detection of Alzheimer's disease. Alzheimers Dement 2015;11:561-578.

56 Piaggi P, Menicucci D, Gentili C, Handjaras G, Gemignani A, Landi A: Singular spectrum analysis and adaptive filtering enhance the functional connectivity analysis of resting state FMRI data. Int J Neural Syst 2014;24: 1450010.

57 Fjell AM, Walhovd KB: New tools for the study of Alzheimer's disease: what are biomarkers and morphometric markers teaching us? Neuroscientist 2011;17:592-605.

58 Smith SJ: EEG in neurological conditions other than epilepsy: when does it help, what does it add? J Neurol Neurosurg Psychiatry 2005 76(suppl 2):ii8-ii12.

59 Cong F, Phan AH, Astikainen P, Zhao Q, Wu $\mathrm{Q}$, et al: Multi-domain feature extraction for small event-related potentials through nonnegative multi-way array decomposition from low dense array EEG. Int J Neural Syst 2013;23:1350006

60 Kimiskidis VK, Kugiumtzis D, Papagiannopoulos S, Vlaikidis N: Transcranial magnetic stimulation (TMS) modulates epileptiform discharges in patients with frontal lobe epilepsy: a preliminary EEG-TMS study. Int J Neural Syst 2013;23:1250035.

61 Herrera LJ, Fernandes CM, Mora AM, Migotina D, Largo R, et al: Combination of heterogeneous EEG feature extraction methods and stacked sequential learning for sleep stage classification. Int J Neural Syst 2013;23: 1350012.

62 Adeli H, Ghosh-Dastidar S, Dadmehr N: A spatio-temporal wavelet-chaos methodology for EEG-based diagnosis of Alzheimer's disease. Neurosci Lett 2008;444:190-194.
63 Ahmadlou M, Adeli H, Adeli A: Fractality and a wavelet-chaos-methodology for EEG-based diagnosis of Alzheimer disease. Alzheimer Dis Assoc Disord 2011;25:85-92.

64 Ghosh-Dastidar S, Adeli H, Dadmehr N: Mixed-band wavelet-chaos-neural network methodology for epilepsy and epileptic seizure detection. IEEE Trans Biomed Eng 2007; 54:1545-1551.

65 Ghosh-Dastidar S, Adeli H, Dadmehr N Principal component analysis-enhanced cosine radial basis function neural network for robust epilepsy and seizure detection. IEEE Trans Biomed Eng 2008;55(2 pt 1): 512-518.

66 Sankari Z, Adeli H, Adeli A: Wavelet coherence model for diagnosis of Alzheimer's disease. Clin EEG Neurosci 2012;43:268-278.

67 Tao H, Zain JM, Ahmed MM, Abdalla AN Jing W: A wavelet-based particle swarm optimization algorithm for digital image watermarking. Int Comput Aided Eng 2012;19:8191.

68 Kodogiannis VS, Amina M, Petrounias I: A clustering-based fuzzy wavelet neural network model for short-term load forecasting. Int J Neural Syst 2013;23:1350024.

69 Cen Z, Wei J, Jiang R: A gray-box neural network-based model identification and fault estimation scheme for nonlinear dynamic systems. Int J Neural Syst 2013;23:1350025.

70 Hsu WY: Single-trial motor imagery classification using asymmetry ratio, phase relation, wavelet-based fractal, and their selected combination. Int J Neural Syst 2013;23: 1350007.

71 Cabessa J, Siegelmann HT: The super-turing computational power of plastic recurrent neural networks. Int J Neural Syst 2014;24: 1450029.

72 Wang Z, Guo L, Adjouadi M: A generalized leaky integrate-and-fire neuron model with fast implementation method. Int J Neural Syst 2014:24:1440004

73 Dai H, Xue G, Wang W: An adaptive wavelet frame neural network method for efficient reliability analysis. Comput Aided Civ Inf 2014; 29:801-814.

74 Alexandridis A: Evolving RBF neural networks for adaptive soft-sensor design. Int J Neural Syst 2013;23:1350029.

75 Adeli H, Ghosh-Dastidar S, Dadmehr N: Alzheimer's disease and models of computation: imaging, classification, and neural models. J Alzheimers Dis 2005;7:187-199; discussion 255-262.

76 Adeli H, Ghosh-Dastidar S, Dadmehr N: Alzheimer's disease: models of computation and analysis of EEGs. Clin EEG Neurosci 2005;36:131-140.

77 Dauwels J, Vialatte F, Cichocki A: Diagnosis of Alzheimer's disease from EEG signals: where are we standing. Curr Alzheimer Res 2010;7:487-505.

78 Zhang C, Wang H, Wang H, Wu MH: EEGbased expert system using complexity measures and probability density function control in alpha sub-band. Int Comput Aided Eng 2013;20:391-405.

79 Rangaprakash D, Hu X, Deshpande G: Phase synchronization in brain networks derived from correlation between probabilities of recurrences in functional MRI data. Int J Neural Syst 2013;23:1350003.

80 Trambaiolli LR, Lorena AC, Fraga FJ, Kanda PA, Anghinah R, Nitrini R: Improving Alzheimer's disease diagnosis with machine learning techniques. Clin EEG Neurosci 2011; 42:160-165.

81 Li D, Xu L, Goodman ED, Xu Y, Wu Y: Integrating a statistical background-foreground extraction algorithm and SVM classifier for pedestrian detection and tracking. Int Comput Aided Eng 2013;20:201-216.

82 Sankari Z, Adeli H, Adeli A: Intrahemispheric, interhemispheric, and distal EEG coherence in Alzheimer's disease. Clin Neurophysiol 2011;122:897-906.

83 Yang AC, Wang SJ, Lai KL, Tsai CF, Yang $\mathrm{CH}$, et al: Cognitive and neuropsychiatric correlates of EEG dynamic complexity in patients with Alzheimer's disease. Prog Neuropsychopharmacol Biol Psychiatry 2013;47: $52-61$.

$84 \mathrm{Hu} \mathrm{M}$, Liang $\mathrm{H}$ : Perceptual suppression revealed by adaptive multi-scale entropy analysis of local field potential in monkey visual cortex. Int J Neural Syst 2013;23: 1350005

85 Al-Jumeily D, Iram S, Hussain AJ, FrancoisBenois V, Fergus P: Early detection method of Alzheimer's disease using EEG signals. Intell Comput Bioinform 2014;8590:25-33.

86 Labate D, La Foresta F, Morabito G, Palamara I, Morabito FC: Entropic measures of EEG complexity in Alzheimer's disease through a multivariate multiscale approach. Sensors J IEEE 2013;13:3284-3292.

87 Waser M, Deistler M, Garn H, Benke T, DalBianco P, et al: EEG in the diagnostics of Alzheimer's disease. Stat Papers 2013;54:10951107.

88 Babiloni C, Del Percio C, Boccardi M, Lizio R, Lopez S, et al: Occipital sources of restingstate alpha rhythms are related to local gray matter density in subjects with amnesic mild cognitive impairment and Alzheimer's disease. Neurobiol Aging 2015;36:556-570.

89 Gallego-Jutglà E, Solé-Casals J, Vialatte FB, Dauwels J, Cichocki A: A theta-band EEG based index for early diagnosis of Alzheimer's disease. J Alzheimers Dis 2015;43:11751184.

90 McBride JC, Zhao X, Munro NB, Jicha GA, Schmitt FA, et al: Sugihara causality analysis of scalp EEG for detection of early Alzheimer's disease. NeuroImage Clin 2014;7:258265

91 Sugihara G, May R, Ye H, Hsieh CH, Deyle E, et al: Detecting causality in complex ecosystems. Science 2012;338:496-500.

92 Cummings JL: Biomarkers in Alzheimer's disease drug development. Alzheimers Dement 2011;7:e13-e44. 
93 NINDS-National Institute of Neurological Disorders and Stroke-Alzheimer's Disease. http://www.ninds.nih.gov/disorders/ alzheimersdisease/alzheimersdisease.htm (accessed January 30, 2015).

94 Francis PT, Palmer AM, Snape M, Wilcock GK: The cholinergic hypothesis of Alzheimer's disease: a review of progress. J Neurol Neurosurg Psychiatry 1999;66:137-147.

95 Ahnaou A, Huysmans H, Jacobs T, Drinkenburg WH: Cortical EEG oscillations and network connectivity as efficacy indices for assessing drugs with cognition enhancing potential. Neuropharmacology 2014;86:362-377.
96 Blurton-Jones M, Spencer B, Michael S, Castello NA, Agazaryan AA, et al: Neural stem cells genetically-modified to express neprilysin reduce pathology in Alzheimer transgenic models. Stem Cell Res Ther 2014;5:46.

97 Chancellor B, Duncan A, Chatterjee A: Art therapy for Alzheimer's disease and other dementias. J Alzheimers Dis 2014;39:1-11.

98 EDU-Characteristics of Learning Disabilities. http://specialed.about.com/cs/ learningdisabled/a/ldmemory.htm (accessed January 29, 2015).
99 NIHs-NINDS Agnosia Information Page. http://www.ninds.nih.gov/disorders/ agnosia/agnosia.htm (accessed January 29, 2015).

100 NLM-Apraxia. http://www.nlm.nih.gov/ medlineplus/ency/article/007472.htm (accessed January 29, 2015), 'Aphasia'. http:// www.nlm.nih.gov/medlineplus/aphasia. html (accessed January 29, 2015).

101 Morabito FC, Campolo M, Labate D, Morabito G, Bonanno L, et al: A longitudinal EEG study of Alzheimer's disease progression based on a complex network approach. Int J Neural Syst 2015;25:1550005. 